\title{
CONFÉRENCE
}

\section{Nanotechnologies et substituts osseux}

\author{
Stéphane Descamps \\ Service de chirurgie Orthopédique et Traumatologique - CHU de Clermont-Ferrand \\ EA 4676 C-Biosenss - Université d'Auvergne \\ descamps.steph@yahoo.fr
}

Les biomatériaux synthétiques sont utilisés en médecine depuis très longtemps, mais ces dernières années, la recherche dans le domaine de ces matériaux a explosé et de nouveaux champs d'exploration sont apparus, telle l'ingénierie tissulaire destinée à offrir aux thérapeutes la capacité de remplacer tout ou partie des tissus malades ou défaillants. Dans le même temps, les travaux de recherche sur le sujet ont progressivement accordé une plus grande importance au concept de bioactivité du matériau à implanter.

De façon parallèle, un champ d'application extrêmement large a été ouvert durant la dernière décénnie au travers des nanotechnologies appliquées à la santé.

Les nanotechnologies permettent par exemple de modifier à l'échelle nanométrique la surface d'un biomatériau pour optimiser l'interaction entre le matériau et son environnement biologique immédiat. Ces nanostructurations de surface ont un intérêt en chirurgie osseuse car elles semblent avoir des fonctions antibactériennes ou de réservoir de molécules actives délivrées in situ. Elles pourraient ainsi améliorer des problématiques cliniques de réponses tissulaires inappropriées au contact des matériaux implantés (inflammation, cicatrisation...). Quelques travaux ont montré l'effet potentiel de modification de nanotopographies de surface sur les réponses cellulaires, avec des applications potentielles d'amélioration d'intégration osseuse de substituts osseux. L'évolution de la chirurgie orthopédique se fait ainsi vers une meilleure prise en compte des problématiques de biologie dans différentes variétés d'implants et des techniques, même s'il n'en reste pas moins vrai que les propriétés mécaniques des implants restent toujours aussi importantes. À ce jour, des études de croissance cellulaires au contact ont été utilisées pour étudier les implants (Yun 2010 ; Brammer 2008 ; Fine 2009 ; Popat 2007 ; Burns 2009 ; Vetrone 2009), mais peu de travaux ont intégré l'étude des propriétés mécaniques des biomatériaux nanostructurés.

Actuellement, seuls quelques produits fondés sur des technologies nano sont disponibles sur le marché Français (Nanogel $^{\circledR}$, Technimed $^{\circledR}$; Nanostim ${ }^{\mathrm{TM}}$, Ostim $^{\circledR}$, Medtronic ; Perossal ${ }^{\circledR}$, AAP Biomaterials ; Vitoss ${ }^{\circledR}$, Scaffold Orthovita, rapport AFSSAPS 2011)

Si le développement de ces nanomatériaux dans le domaine médical semble prometteur, il n'en reste pas moins que leur utilisation suscite de nombreuses interrogations dans le domaine de leur potentielle toxicité.

Le monde des nanostructures se présente sous deux formes fondamentalement très distinctes : les nanoparticules qui sont des entités libres, et les nanostructurations de surface qui sont des modifications structurales de la surface d'un matériau. Si la toxicité des nanoparticules fait à juste titre l'objet d'une actualité prolifique et est à l'origine de travaux de recherche visant à étudier son innocuité, il n'en va pas de même pour les nanostructurations des surfaces possiblement destinées à être utilisées en clinique. A notre connaissance, cet aspect très spécifique de toxicité des nano-surfaces ne fait l'objet que de peu de travaux de recherche. Or les conditions même d'utilisation de ce type de matériaux dans les milieux biologiques posent un certain nombre de questions quant à leur stabilité physique et chimique, à leur toxicité propre et à leurs produits de relargage ou de dégradation. 